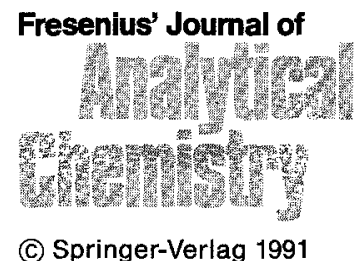

\title{
Erratum
}

Fresenius J Anal Chem (1991) 337:220-224

\section{History of the relationship chemistry - mathematics}

\section{Antonio Drago}

Department of Physical Sciences, Section of History of Physics, University of Naples, Mostra d'Oltremare, I-80125 Napoli, Italy

The scheme on p. 221 (top of first column) should correctly be as follows:

ADEQUACY OF ANY KIND OF MATHEMATICS

TO PHYSICAL AND CHEMICAL THEORIES

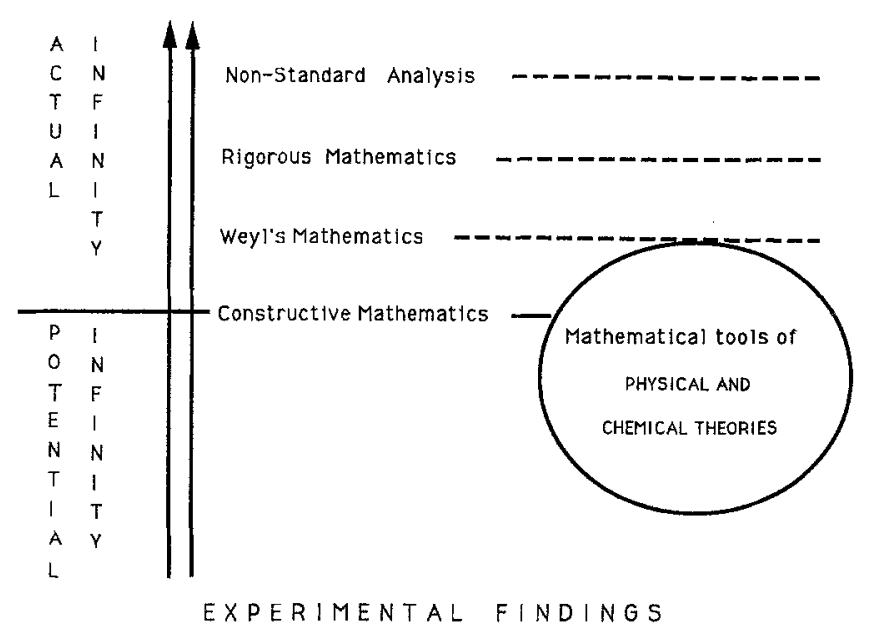

\section{Experimental findings}

The mathematical tools of physical and chemical theories are derived by abstraction processes from experience. Constructive mathematics, inasmuch it is confined to the use of potential infinity only, includes only a part of the set of such mathematical tools. Instead, the same set is fully included by Weyl's elementary mathematics, operating at a minimum level of actual infinity; then, at higher levels, it is included by rigorous mathematics and by non-standard analysis. 